\title{
Effect of Solution Temperature on the Structure of Thioacetyl-terminated Tolane Self-assembled Monolayers on Au(111)
}

\author{
Youngdo Jeong, Jin Wook Han," Changiin Lee, and Jaegeun Noh*

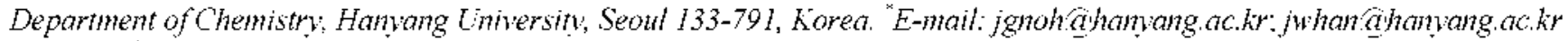 \\ Actranced Material Dision, Korea Research Institute of Chemical Technologn, Daejeon 305-600, Korea \\ Recerved I Farch 30, 2008
}

\begin{abstract}
Key Words : Self-assembled monolayers. Tolanemethylthioacetate. Temperature effect. Scanning tunneling microscopy
\end{abstract}

Self-assembled monolayers (SAMs) formed by organosulfur compounds on metal surfaces offer a simple and powerful route for the fabrication of functional ultrathin films that can be employed in various technical applications such as corrosion inhibition. chemical sensors. biosensors. nanopatterning, and molecular electronic devices. ${ }^{1.7}$ Recently. SAMs derived from conjugated aromatic thiols on gold have attracted nuch attention as a result of their interesting electrical and optical properties as well as their potential for use in molecular electronics applications. ${ }^{7010}$ Oligo(phenylene ethylene) (OPE) molecules have been considered as an extremely promising molecular sy stem for device applications because they have a $\pi$-conjugated and rod-like molecular structure. The formation of OPE SAMs and their electrical properties have been extensively characterized by various surface characterization tools. ${ }^{11.12}$ Thioacetyl-protected conjugated molecules with a high chemical stability have been frequently used for electronic device applications. ${ }^{7.11}$.12 It has been reported that these molecules can form chenisorbed SAMs on gold in acid- or base-catalyzed mediunts ${ }^{11.11}$ or without any catalyzed medium. ${ }^{13-16}$ Prior to the development of SAM-based devices, it is essential to understand the formation and structure of SAMs on gold. However, although several macroscopic measurements for SAMs formed by thioacetyl-terminated molecules have been carried out to gain insight into SAM formation. there have been only a few nanometer-scale reports on these SAMs.

Among these previous reports. one study showed that the conductance switching behavior in single molecules is due to conformational changes of molecular backbones. rather than electrostatic effects of charge transfer. ${ }^{1 ;}$ Therefore. for SAM-based device applications. it is very important to control the two-dimensional structure of conjugated SAMs as well as to obtain high-quality SAMs. As far as processing conditions go. it is well known that the thermal annealing of pre-covered alkanethiol SAMs at $70-100^{\circ} \mathrm{C}$ results in the formation of large ordered domains and healing of vacancy islands (VIs) ${ }^{17}$ It was also found that alkanethiol SAMs with large well-ordered donains and few VIs can be fonned by liquid and vapor phase deposition methods at high temperature as a one-step process to obtain high quality SAMs. ${ }^{\text {is }}$

Tolanemethylthioacetate (TMTA) with a $\pi$-conjugated. rigid molecular backbone is one of interesting molecules for electronic device application. Figure I shows the structural formula of TMTA molecule. In this study, to obtain highquality ordered TMTA SAMs on Au(111), we examine the effect of solution temperature on the formation and structure of TMTA SAMs using STM. We report herein the first STM results showing the phase transition from the disordered phase to the ordered phase of TMTA SAMs as solution temperature increased.

TMTA was synthesized by modifying a previously reported method. ${ }^{19} \mathrm{Au}(11 \mathrm{l})$ substrates on mica were prepared by vacuum deposition as previously described. "The SAMs were prepared by inmersing Au(111) substrates in $0.5 \mathrm{mM}$ $\mathrm{N}, \mathrm{N}^{\prime}$-dimethylformamide (DMF) solutions of TMTA at room temperature, $50^{\circ} \mathrm{C}$, and $80^{\circ} \mathrm{C}$ for $24 \mathrm{~h}$. STM measurements were carried out using a NanoScope $\mathrm{E}$ with a commercial Pt/ Ir (80:20) tip under ambient conditions.

The STM inages in Figure 2 show the surface structures of TMTA SAMs on Au(111) formed after immersion as a function of solution temperature. Clearly. the solution temperature markedly affects the formation and structure of TMTA SAMs on $\mathrm{Au}(111)$. Although acetyl-protected TMTA molecules on Au(111) form chemisorbed SAMs via the spontaneous deprotection of acetyl group during the adsorption of TMTA in DMF solution. ${ }^{i z}$ TMTA molecules form disordered phases containing partially ordered domains and molecular aggregates at room temperature, as shown in Figure $2 a$. Differently from the formation of well-ordered SAMs by alkanethiols at room temperature. TMTA molecules do not form highly ordered SAMs. The unusual structural characteristics of TMTA SAMs can be ascribed to the lower chemical activity of sulfur headgroups against the gold atoms. which is due to the existence of an electron withdrawing acetyl group attached to the sulfur atom. In contrast, more ordered SAMs with a uniform surface morphology were formed in the medium-temperature solution of $50^{\circ} \mathrm{C}$, as shown in the STM inage of Figure $2 \mathrm{~b}$. We observed a large number of

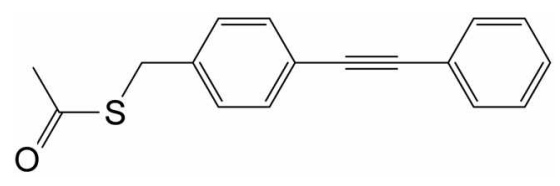

Figure 1. Structural fomula of TMTA. 

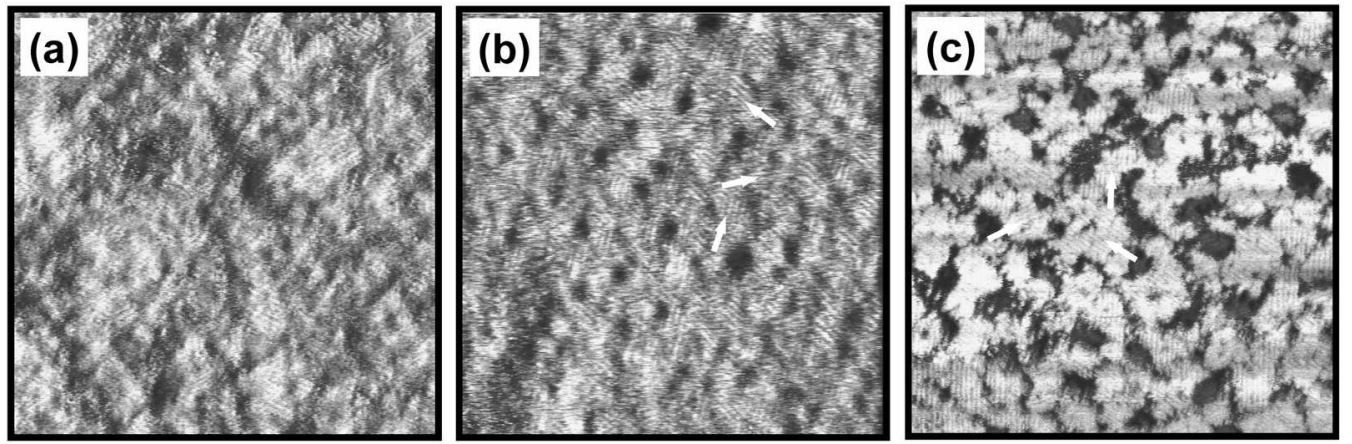

Figure 2. STM images of TMTA SAMs on Au( 111$)$ formed in a $0.5 \mathrm{mM}$ DMF solution after immersion for 24 h as a function of solution temperature: (a) room temperature, (b) $50^{\circ} \mathrm{C}$, and (c) $80^{\circ} \mathrm{C}$. Scan sizes were (a) $100 \mathrm{~nm} \times 100 \mathrm{~nm}$, (b) $100 \mathrm{~nm} \times 100 \mathrm{~nm}$, and (c) $120 \mathrm{~nm} \times$ 12011112.

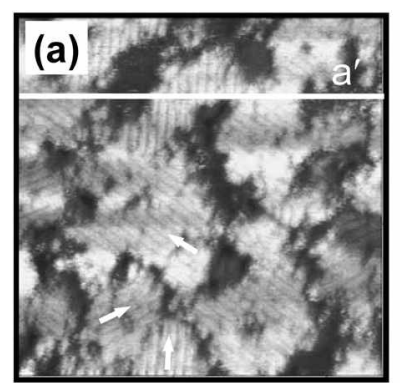

(b)

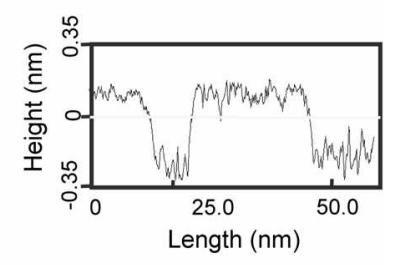

Figure 3. (a) A 60 non $\times 60$ min STM image of TMTA SAMs on Au( 111$)$ tormed at $80^{\circ} \mathrm{C}$. This image clearly shows two-dimensional ordered SAMs with three domain orientations. (b) Crosssectional profile along line a on the STM image shows a height difference between the ordered domains and the dark regions.

small size ordered domains with three domain orientations (see arrows) ranging from several nun to $12 \mathrm{~lm}$, and small size Vls (dark holes) with a monatomic step height of 0.25 $1 \mathrm{~nm}$ that are known to appear during chemisorption of organosulfur compounds on gold surfaces. ${ }^{17.18}$ Conipared to the STM inage of Figure $2 b$, the STM image in Figure $2 c$ (TMTA SAMs on $\mathrm{Au}(111)$ formed at $80^{\circ} \mathrm{C}$ ) obviously shows crystallized ordered domains with three domain orientations and clear VIs irregular in shape. The domain sizes were measured to be approximately $15-25 \mathrm{~nm}$. and are almost twice the size of those observed in SAMs formed at $50^{\circ} \mathrm{C}$. From this study, we report for the first time phase transitions of TMTA SAMs on Au(111) from the disordered phase to 2D ordered phases with increasing solution temperature.

The magnified STM image of Figure 3a clearly shows a high degree of structural order of TMTA SAMs on Au(111) formed at $80^{\circ} \mathrm{C}$. Interestingly. the ordered domains. consisting of molecular rows with pairs of molecules. are mainly separated by a network of connected VIs that has never been observed in conventional alkanethiol SAMs. However. at present. it is hard to understand the origin of such structural behaviors of TMTA SAMs. The distance between the molecular rows was $1.4 \mathrm{~nm}$. The height profile along line $a^{\prime}$ of the STM image (Figure 3b) shows that the height differences between the ordered domains and the dark regions are $0.22-0.27 \mathrm{~nm}$. which is very similar to the monatomic depth of $0.25 \mathrm{~mm}$. Based on this observation, the dark regions can be regarded as VIs formed as a result of the formation of chemisorbed SAMs.

In summary, we have clearly demonstrated that solution temperature strongly affects the formation and 2D molecular arrangements of TMTA SAMs on gold. Moreover, we can obtain TMTA SAMs with a ligh degree of structural order in high-temperature solutions. We also found that the growth of thioacetyl-terminated tolane SAMs on gold is remarkably different from that of organic SAMs.

\section{References}

1. Love. T. C.: Estroff. L. A.: Kriebel. T. K.: Nizo. R. G.: Whitesides. G. M. Chem. Ren. 2005, 105, 1103.

2. Crook. R. M.: Ricco. A. J. Acc. Chem Res. $1998,31,219$

3. Noh, J.; Jeong, Y.; Ito, E.: Hara. M. J. Plns. Chem. C 2007, 111. 2691

4. Noh. J.: Kato. H. S.: Kawai. M.: Hara. M. J. Phns Chem. B 2006. 110.2793.

5. Noh. T.: Park. H.: Jeong. Y.: Kwon. S. Bull. Korean Chem. Soe. 2006. 27,403

6. Hlood. A. H: Stoddart. J. F.: Steuerman. D. W.: Heath. J. R. Science 2004. 306,2055

7. Tour. T. M. Ace. Chent. Res. 20M0.33. 791

8. Ramachandran. G. K.: Hopson. I. J.: Rawlett. A. M.: Nagahara. L. A.: Primak. A.: Lindsay. S. M. Science 2003. 300. 1413.

9. Seminario, J. M.: Zacarias, A. G: Tour, J. M. J. Phus. Chem. A 1999. 103,7883.

10. Donhauser. Z. J.: Mantooth. B. A; Kelly: K. H.: Bumn, L. A. Monnell. J. D.: Stapleton. J. J.: Price. L. W.. Ir:: Rawlett. A. M.: Allara. D. L.: Tour. T. M.: Weiss. P. S. Science 2001. 292. 2303.

11. Chet1. L.: Yang. J.: Yao. Y.: Price. L) W. Jr: Lirk. S. M.: Tour. J. M. Langmiir 2004. 20, 1335 .

12. Blum. A. S.: Yang, J. C.: Shashidhar, R.: Ranta B. Appl. Phys. Lett. $2003,82.3322$.

13. Jeong. Y.: Lee. C.: Ito. E.: Hara. M.: Noh. J. Jpn. J. Appl. Plns 2006. +5.5906

14. Jeong. Y.: Han1. I. W.: Kim. N.: Lee. Y.: Lee. C.: Hara. M.: Noh. J. Bull. Korean Chem. Soc, 2007, 28. 2445.

15. Jeong. Y; Kwon, S.; Kang, Y.: Lee. C.: Ito. E.: Hara, M; Noh, J. Ultramicroscopy, 2007. 107, 1000 .

16. Jeong. Y: Chung. H.: Noh. J. Colloid and Surface A: Phwicochem. Eing Aspects 2008. 313-31t. 608 .

17. Poirier. G. E. Chent Rev 1997.97. 1117.

18. Kwon. S.; Choi. J.; Lee, H.: Noh, J. Colloid and Surface A: Phusicochem. Eng. Aspects 2008. 313-31t, 324.

19. Huang. S. H.: Tour. J. M. J. Org Chem. 1999. 64, 8898. 